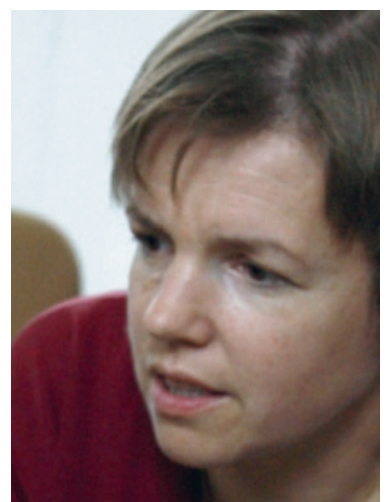

Dr. sc. nat. Beatrix Falch, Zürich

\section{Ganzheitliches Therapiekonzept in der Geriatrie}

Der Anteil der Menschen, die über 60 Jahre alt sind, nimmt kontinuierlich zu. Daher überrascht es nicht, dass auf diese Altersgruppe ein besonderes Augenmerk gerichtet wird; so auch in dieser Ausgabe der SchweIzerische ZeitSCHRIFT FÜR GANZHEITSMEDIZIN.

Die gesundheitlichen Probleme älterer Menschen sind vielfältig. Es gilt dabei zu bedenken, dass neben chronischen Grunderkrankungen eine Reihe von Faktoren, die die Lebensweise betreffen - wie beispielsweise die Ernährung, die körperliche Aktivität oder die Beteiligung am gesellschaftlichen Leben -, einen entscheidenden Einfluss auf die Lebensqualität dieser Patientengruppe haben. Hinzu kommt, dass sich der Stoffwechsel im Laufe des Lebens verändert und dies vor allem bei Betagten bezüglich der Dosierung und dem Interaktionspotenzial von Medikamenten berücksichtigt werden muss. Ältere Menschen sind also eine Altersgruppe, die nicht nach Schema «F» behandelt werden kann.

Untersuchungen haben gezeigt, dass die Ernährung Betagter oft unausgewogen ist. Dies hat häufig damit $\mathrm{zu}$ tun, dass viele ältere Menschen alleine leben. Ohne Gesellschaft macht das Essen weniger Freude, und die Motivation, sich selber etwas zu kochen, ist nicht sehr gross. So ist der Appetit bei alleinstehenden Älteren meist reduziert, und es wird die Nahrung bevorzugt, die schnell und einfach zubereitet werden kann. Auch die altersbedingte veränderte Geschmacksempfindung beeinflusst das Essverhalten.
Hinzu kommt der Bewegungsmangel. Haben ältere Patienten in den Beinen und Gelenken Schmerzen, vermeiden sie eher eine sportliche Betätigung. Aber auch depressive Verstimmungszustände begünstigen die körperliche Inaktivität. Die Motivation, sich zu bewegen, hängt zum Grossteil davon ab, ob man Freunde oder Bekannte hat, mit denen man zusammen Sport treiben kann.

Gründe, seinen Lebensstil nicht zu ändern, gibt es scheinbar viele. Es ist schon fast wie in einem Teufelskreis: Lebt man als Betagte/r alleine, fühlt man sich einsam und depressive Verstimmungen sind nicht selten. Das führt zu einer Lustlosigkeit und Demotivation, sich ausgewogen zu ernähren und zu bewegen sowie überhaupt irgendeine Initiative zu ergreifen. Dies wiederum hat gesundheitliche Beeinträchtigungen zur Folge, die auf die Stimmung drücken.

Und dabei wäre es doch gar nicht so schwer, Abhilfe zu schaffen. Ernährungsratgeber, Seniorengymnastik und eine Vielzahl von naturheilkundlichen Methoden stehen zur Verfügung, um das physische wie psychische Wohlbefinden von Betagten positiv zu beeinflussen. Kann man mit diesen Massnahmen das Wohlbefinden von Betagten steigern und deren Selbstständigkeit erhalten, hat man schon viel erreicht.

Über diese möglichen naturheilkundlichen Methoden, mit denen Betagte befähigt werden, besser im Alltag alleine zurechtzukommen, die aber leider in der Praxis noch unterschätzt werden, können Sie in diesem Heft mehr erfahren.

Dass eine Therapie nur etwas nützt, wenn man sie auch anwendet, ist eine Binsenwahrheit. Aber genau hier scheitern viele therapeutische Ratschläge. Denn trotz der erklärten Evidenz fehlt den Betagten die Motivation, ihre Ernährung zu ändern oder sich mehr bzw. gezielt zu bewegen. Wie man ältere Patienten motivieren kann, beispielsweise an einem Bewegungsprogramm zur Prävention von Stürzen teilzunehmen, können Sie ebenfalls in den «Im Fokus»-Beiträgen nachlesen.

Welche der verschiedenen Therapieoptionen soll man nun aber wählen? Es gilt festzuhalten, dass es nicht um «entweder die eine oder die andere Therapie» geht, sondern um eine sinnvolle Kombination, wie dies auch das Expertengespräch in diesem Heft aufzeigt. Die (Heil-)Kunst besteht darin, adäquate Therapien und auf die einzelne Person abgestimmte Therapiekombinationen auszuwählen, um einerseits den erwünschten therapeutischen Effekt $\mathrm{zu}$ erzielen und andererseits die Nebenwirkungen auf

\section{KARGER}

Fax +497614520714 Information@Karger.de www.karger.com
Dr. sc. nat. Beatrix Falch

Hochstrasse 51, 8044 Zürich, Schweiz

bfalch@sunrise.ch 
einem möglichst niedrigen Niveau zu halten. Und nicht alle Therapien können kombiniert werden. Wenn man beispielsweise Phytotherapie und Schulmedizin gleichzeitig einsetzt, dann muss man sehr genau wissen, ob und wie eine pflanzliche Zubereitung die Pharmakokinetik eines schulmedizinischen Präparates beeinflusst. Ohne Erfahrung und ohne eine fundierte Ausbildung kann keine ganzheitliche Therapie betrieben werden. Eine ganzheitliche Therapie ist jedoch nötig - egal in welchem Alter! Jeder wünscht sich, gesund und geistig fit alt zu werden. Die medizinischen Fortschritte wollen uns glauben machen, dass wir tatsächlich das «Altwerden» im Griff haben und das Bild der buckeligen und runzeligen Alten der Vergangenheit angehört. Dabei blenden wir oft aus, dass in unserer Gesellschaft Gesundheit auch etwas mit Wohl- stand zu tun hat. Nur wer sich eine Unterstützung im Haushalt, Bewegungstrainings, Massagen, Wellnesskuren und die von den Krankenkassen (noch) nicht bezahlten naturheilkundlichen Therapien leisten kann, kann trotz Grunderkrankungen mit einem hohen Mass an Lebensqualität alt werden.

Wer mit betagten Menschen zu tun hat, wird sehr schnell gewahr, dass alte - wie ganz junge - Menschen betreut und begleitet werden müssen, aber nicht bevormundet werden dürfen. Man muss auf die individuellen Bedürfnisse der älteren Menschen eingehen und ihre Lebenserfahrung nutzen und schätzen können. Dies ist durchaus eine Herausforderung, der sich nicht nur die Medizin, sondern die ganze Gesellschaft stellen muss. 